\title{
Full Speed in der Neuroradiologie!
}

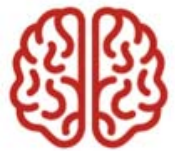
DGNR

Deutsche Gesellschaft für Neuroradiologie e.V.

Liebe Kolleginnen und Kollegen,

im Namen der VMTB - Vereinigung medizinisch-technischer Berufe in der DRG - begrüBen wir Sie herzlich zum MTRA-Programm auf der neuroRAD-Jahrestagung 2018 am neuen Kongressstandort Frankfurt am Main!

Von Donnerstag, 4. Oktober bis Samstag, 6. Oktober erwartet die MTRA-Community ein volles Programm: Full Speed in der Neuroradiologie, wie es einer der Vorträge auf den Punkt bringt!

Neben den fachlich-medizinischen Themen, wie der Stroke-Behandlung (Was bedeuten die bunten Bilder in der PerfusionsCT?; Freitag, 5.10.) und der Kinderneuroradiologie (Babys in der Neuroangiografie; Freitag, 5.10.), werden Themen um den MTRA-Beruf herum, wie z. B. Stressmanagement und Umgang mit psychischer Belastung (Freitag, 5.10.), vorgestellt.

Gemäß dem Kongressmotto neuroRAD goes international wird sich eine Session der Frage widmen, wie man als MTRA im Ausland arbeiten kann (Freitag, 5.10.).

Highlight des 3-tägigen MTRA-Programms ist am Samstag, dem 6.10., die Stroke-Rallye - ein Hands-on zur neuroradiologischen Akutversorgung beim Schlaganfall mit anschließender Preisverleihung.

Erstmalig wird in diesem Jahr ein Programm für den MTRA-Nachwuchs angeboten. Der MTRA-Schülertag 2018 unter der Schirmherrschaft der VMTB findet am Donnerstag, dem 4. Oktober von 9-17 Uhr statt und bietet den Teilnehmern vielfältige Gelegenheit, die MTRA-Arbeit in der Neuroradiologie kennenzulernen - mit Vortragstiteln die neugierig machen: „Neuroananatomie für Dummies“, „Schatz, ich hab Migräne“,
„Die drei ??? und die Kammer der Rätsel: Implantate, Körperschmuck und anderes Blech im MRT“, um nur ein paar Vortragsthemen zu nennen. Seien Sie bei der Premiere neuroRAD goes international in Frankfurt a. M. dabei! Begleiten Sie uns durch ein interessantes Vortragsprogramm!

Claus Becker

Vorsitzender der VMTB

Michael Wiertz

Lehr-MTRA UK Aachen

Annette Saur und Dorothe Krug

kooptierte Mitglieder der VMTB

\section{Einladung des \\ Kongresspräsidenten}

Sehr geehrte Damen und Herren, liebe Kolleginnen und Kollegen,

vom 3. bis zum 6. Oktober 2018 findet in Frankfurt am Main die 53. Jahrestagung der Deutschen Gesellschaft für Neuroradiologie statt.

Mit dem Wechsel des Standortes haben wir das Programm erheblich ausgeweitet und internationalisiert.

Es erwartet Sie

- ein durchgängiges Fortbildungs- und Refresher-Programm, auch für Weiterbildungsassistenten (Fit für Facharzt),

- wissenschaftliche Vorträge eingerahmt von einem themenbezogenen Update durch erfahrene Referenten,

- die German-Stroke-School mit Training an Mentice-Simulatoren (begrenzte Kapazitäten, frühzeitige Anmeldung erforderlich!),

- ein englischsprachiges Programm mit Top-Referenten und Themen aus Diagnostik und Intervention,

- Spezialkurse wie die Zertifizierungskurse nach DGNR und DeGIR, fMRT- und Spektroskopie-Kurse, Fachkunde-Aktualisierung u. a.m. sowie

- Keynote-Lectures an allen Hauptkongresstagen!
Wir freuen uns auf Ihren Besuch!

Prof. Dr. Martin Wiesmann

Kongresspräsident 2018

\section{Keynotes an allen}

Kongresstagen!

An allen Haupttagen des Kongresses (Donnerstag bis Samstag) werden in diesem Jahr Keynotes abgehalten. Neben Größen aus der Neuroradiologie und der angrenzenden Neurobiologie dürfen Kongressbesucher gespannt sein auf spannende Einblicke fachfremder Referenten:

\section{Donnerstag, 4.10.2018,} 12.30 - 13:15 Uhr

Dr. Matthias Münzberg/Martin Egerth (Lufthansa): „Safety First - Wenn Krankenhäuser wie Flugzeuge wären“

\section{Donnerstag, 4.10.2018, 17.15 - 18:30 Uhr}

Keynote-Lecture: Prof. Dr. Daniela Prayer „Brain plasticity- lessons from fetal MRI“/ Andrea Rossi „Imaging of Leukodystrophies"

\section{Freitag, 5.10.2018,} 11:15 - 12:30 Uhr

Prof. Dr. Ingo Bechmann „Anatomie-Update Hirnhäute, Liquor-System und perivaskuläre Räume“ / Dr. Anne G. Osborn „Imaging and Pathology of Perivascular Spaces“

Freitag, 5.10.2018, 17:00 - 18:30 Uhr

Keynote-Lecture: Dr. Anne G. Osborn „Brain in Flame“

\section{Samstag, 6.10.2018, 10:15 - 11:00 Uhr}

Keynote-Lecture: Prof. Dr. Dr. Onur Güntürkün „Kognition ohne Kortex“

Alle Informationen zum Programm und Online-Anmeldung unter www.neurorad.de/programm.html Ermäßigung für Mitglieder der DRG kostengünstiges Kombiticket für Neumitglieder 


\section{Einladung und \\ Tagesordnung}

Zur Mitgliederversammlung der Deutschen Gesellschaft für Neuroradiologie e. V. Freitag, 5.10.2018, 18.45 - 19.45 Uhr Saal Horizont / Kap Europa Frankfurt am

Main

- TOP 1: Begrüßung und Feststellung der Beschlussfähigkeit

- TOP 2: Genehmigung der Tagesordnung

- TOP 3: Genehmigung des Protokolls vom 12.10.2017 in Köln

- TOP 4: Totengedenken

- TOP 5: Bericht des Präsidenten Prof. Arnd Dörfler

- TOP 6: Bericht des Schatzmeisters Prof. Jens Fiehler

- TOP 7: Bericht der Kassenprüfer

- TOP 8: Entlastung des Vorstands

- TOP 9: Bekanntgabe neuer Mitglieder

- TOP 10: Berichte aus den Ausschüssen

- TOP 11: Bericht des Kongresspräsidenten 2018

- TOP 12: Verabschiedung der Satzung in der Neufassung vom 01.07.2018

- Top 13: Wahl des DGNR-Präsidenten $2020-2022$

- TOP 14: Wahl des Schatzmeisters 2018-2020

- TOP 15: Wahl des Schriftführers 2018 2020

- TOP 16: Wahl der Ehrenmitglieder 2019

- TOP 17: Ausblick auf die 54. Jahrestagung der DGNR von Donnerstag, 10. Oktober 2019 bis Samstag, 12. Oktober 2019 in Frankfurt (Prof. Dr. Claus Zimmer)

- TOP 18: Verschiedenes

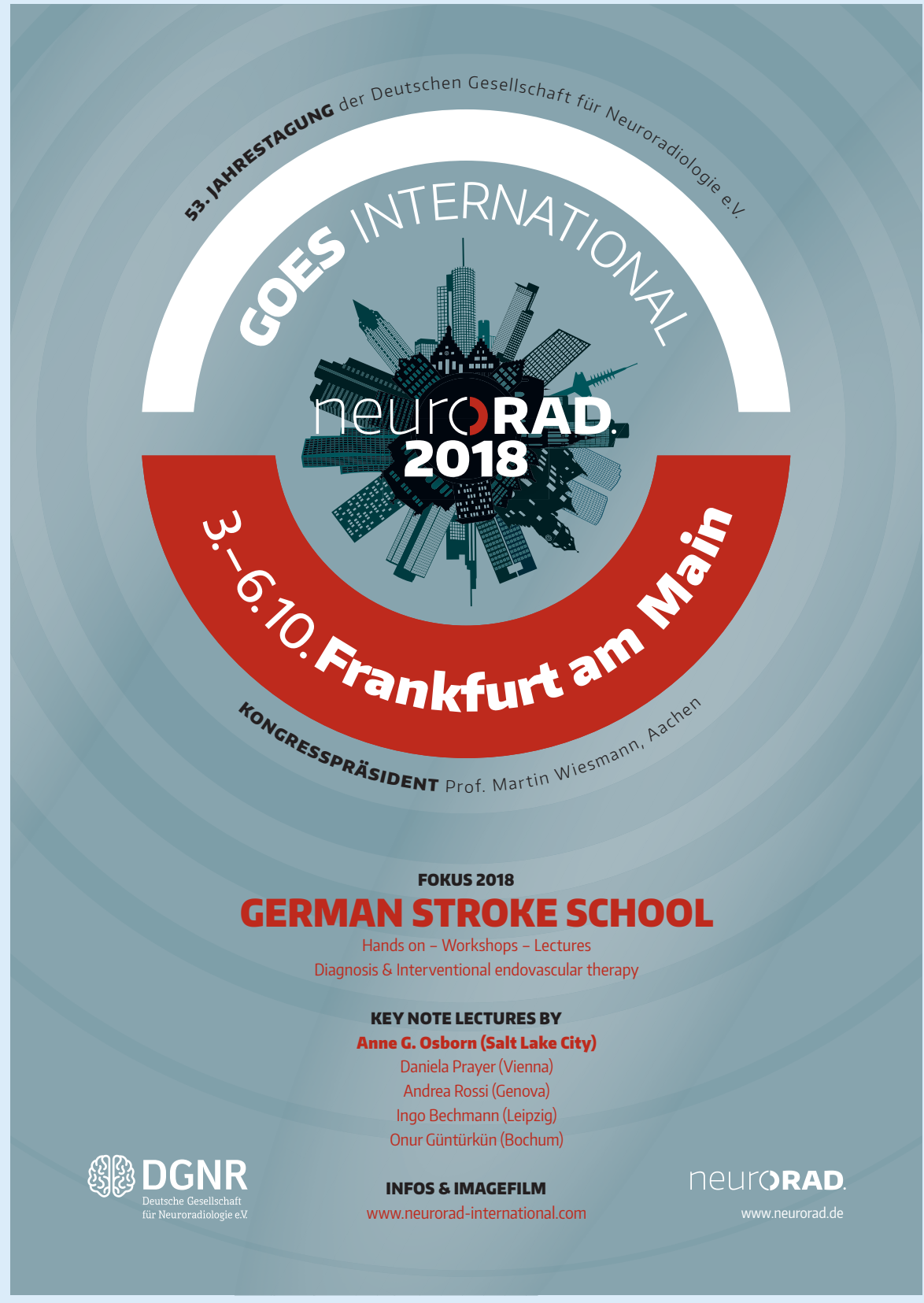

Plakat der neuroRAD-Jahrestagung 2018 @ DGNR 